\title{
Angioedema ou síndroma da veia cava superior: relato de caso
}

João Vieira Antunes,* Beatriz Cordeiro Aguiar,** Margarida Sampaio Leite***

\section{RESUMO}

Introdução: O médico de família (MF) depara-se regularmente com sinais e sintomas comuns que podem ser a tradução de patologias de curso menos benigno. Com este caso pretende-se demonstrar como o achado relativamente comum de edema facial pode ser interpretado erroneamente como angioedema, quando a patologia subjacente é a síndroma da veia cava superior (SVCS).

Descrição do caso: Homem, 61 anos, recorreu ao MF por história de síncope. Foi enviado ao serviço de urgência (SU) hospitalar, onde foi assumido o diagnóstico de síncope vasovagal e detetada anemia microcítica hipocrómica. O estudo da anemia revelou ferropenia e uma úlcera no antro gástrico. Passados três meses voltou à Unidade de Saúde Familiar por edema facial, tendo sido medicado com anti-histamínico. Por persistência das queixas, recorreu ao SU onde foi feito o diagnóstico de angioedema. Um mês depois foi internado para tratamento de uma hemorragia digestiva alta com origem na úlcera gástrica. Voltou posteriormente ao SU por queixas respiratórias, onde se efetuou telerradiografia torácica que revelou alargamento do mediastino superior. No internamento diagnosticou-se um linfoma B que terá condicionado compressão da veia cava superior. Comentário: A dificuldade em diferenciar angioedema de SVCS revelou-se fulcral no desenvolvimento do presente caso. Situações de confusão diagnóstica entre estas duas entidades são já descritas na literatura. Questões relativas à complexidade das apresentações clínicas e às dificuldades sentidas pelo MF na gestão do presente caso serão discutidas.

Palavras-chave: Linfoma; Angioedema; Síndroma da Veia Cava Superior.

\section{INTRODUÇÃO}

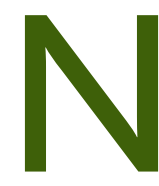

a prática clínica diária, o médico de família (MF) depara-se regularmente com sinais e sintomas comuns e de aparente benignidade que, não raras vezes, são a tradução de patologias menos prováveis e de curso pouco indolente.

$\mathrm{O}$ angioedema caracteriza-se pelo extravasamento vascular que ocorre na derme e tecido subcutâneo da face, lábios e língua, atingindo ocasionalmente as vias aéreas superiores, mãos, pés ou até mesmo a mucosa gastrintestinal. Pode ter causas diversas, nomeadamente alérgicas, genéticas, autoimunes ou medicamentosas, e assemelhar-se a outras patologias, entre

*Médico Interno de Medicina Geral e Familiar. USF Brás Oleiro, ACES Porto II, Gondomar.

**Médica Interna de Medicina Geral e Familiar. USF Brás Oleiro, ACES Porto II, Gondomar.

***Médica Interna de Medicina Geral e Familiar. USF Brás Oleiro, ACES Porto II, Gondomar. elas, a síndroma da veia cava superior (SVCS), entidade ameaçadora da vida e uma emergência médica. ${ }^{1}$ Poucos estudos de prevalência relativos ao angioedema estão reportados a nível europeu. Um estudo dinamarquês estimou uma prevalência de $7,4 \%$ da doença na população em geral, alertando para a possibilidade deste valor poder subestimar o verdadeiro número de casos existentes. ${ }^{2}$ Nos Estados Unidos estima-se que o angioedema e a urticária atinjam cerca de 14 a 25\% da população, sendo que estas entidades clínicas são frequentemente diagnosticadas pelo $\mathrm{MF}^{3}$

A SVCS ocorre quando há uma obstrução do retorno venoso da cabeça, pescoço e das extremidades superiores devido a uma compressão externa ou obstrução intrínseca da veia cava superior. De entre os principais fatores etiológicos relacionados com a SVCS destacam-se as doenças malignas (entre elas, o linfoma) e as causas trombóticas. ${ }^{4-5}$ 
No presente caso é relatada a história de um homem que recorreu ao seu MF por um episódio de síncope, assumida, quanto à sua etiologia, como síncope vasovagal. Posteriormente, achados clínicos como a existência de uma anemia microcítica hipocrómica, que se associou à ocorrência de hemorragia digestiva alta (HDA) por úlcera gástrica e, mais tarde, o surgimento de edema facial, tornaram complexa a integração dos sintomas do doente e difícil a identificação do problema de saúde subjacente. O difícil esclarecimento da etiologia do edema facial, que levou à confusão diagnóstica entre angioedema e SVCS, e a dificuldade de articulação com as consultas de especialidade hospitalar foram fatores que atrasaram o diagnóstico do linfoma B que se veio a descobrir.

\section{DESCRIÇÃO DO CASO}

O presente caso relata a história de um homem de 61 anos, operário de construção civil, de raça caucasiana, residente num concelho do noroeste do país. É viúvo, tem um filho de 35 anos e vive sozinho, sendo autónomo para as atividades da vida diária.

Trata-se de um doente com antecedentes patológicos de diabetes mellitus tipo 2, dislipidemia e hipertensão arterial, doenças devidamente controladas com metformina e vildagliptina (1.000mg + 50mg bid), sinvastatina $(40 \mathrm{mg}$ id), candesartan e hidroclorotiazida (16mg + 12,5mg id), respetivamente. Tem hábitos etílicos (100g álcool/dia), não apresenta hábitos tabágicos, desconhece alergias e não apresenta antecedentes familiares de relevo.

Recorreu, em agosto de 2013, a uma consulta aberta na sua Unidade de Saúde Familiar (USF) pois, ao levantar-se nessa manhã, teve tonturas e perdeu a consciência, tendo caído na altura. Negava outra sintomatologia prévia à queda, nomeadamente palpitações, precordialgia, náuseas, vómitos ou queixas prodrómicas. Ao exame objetivo apresentava tensão arterial mais baixa que o habitual $(100 / 60 \mathrm{mmHg})$, glicemia capilar dentro dos parâmetros normais $(114 \mathrm{mg} / \mathrm{dl})$ e uma pequena escoriação frontal direita. A auscultação cardiopulmonar e o exame neurológico não apresentavam alterações de relevo. Assumido o diagnóstico de síncope, foi enviado ao serviço de urgência (SU) hospitalar, onde efetuou tomografia computorizada (TC) cerebral e eletrocardiograma, que se revelaram normais. No estudo analítico foi objetivado um valor de hemoglobina $(\mathrm{Hb})$ de $10,6 \mathrm{mg} / \mathrm{dl}$, associado a microcitose e hipocromia. Teve alta hospitalar com diagnóstico de síncope de provável etiologia vasovagal.

Em nova consulta na USF (30/08/2013) foi considerado não haver indicação para prosseguir o estudo da etiologia da síncope, uma vez que se tratava do primeiro episódio e sem sintomas associados. O estudo da anemia englobou investigação analítica, endoscopia digestiva alta (EDA) e colonoscopia total. Estes exames revelaram ferropenia (ferritina: $9,7 \mathrm{ng} / \mathrm{mL}$ ) e uma úlcera escavada de $20 \mathrm{~mm}$ do antro distal com bordos congestivos e histologia positiva para Helicobacter pylori (Hp), sem evidência de malignidade (a colonoscopia não apresentava alterações). Foi instituída terapêutica tripla de erradicação do Hp e iniciada suplementação com ferro oral (proteinossuccinilato de ferro 800 $\mathrm{mg} / 15 \mathrm{ml}$ id), tendo os valores de $\mathrm{Hb}$ subido para o limite inferior da normalidade após um mês de terapêutica.

Em novembro (29/11/2013), o doente voltou a recorrer ao seu MF, desta vez por aparecimento de edema facial de agravamento matinal, com cerca de três dias de evolução, sem prurido, edema da língua ou dispneia. Negava história prévia de reações alérgicas alimentares e medicamentosas, utilização de produtos de higiene ou cosmética diferentes dos habituais e ocorrência de picada de inseto. Ao exame objetivo apresentava-se eupneico, com mucosas descoradas, fácies ligeiramente pletórica e discreto edema peri-orbitário e malar, sem outros achados relevantes. Não havendo sinais de alarme, o episódio foi interpretado como uma provável reação alérgica, tendo sido medicado com bilastina (20mg id) e agendada consulta para reavaliação.

Uma semana depois $(02 / 12 / 2013)$ recorreu ao SU hospitalar por agravamento do edema facial. Efetuou telerradiografia torácica e estudo analítico, que não mostraram alterações. Apresentou melhoria após tratamento com hidrocortisona e clemastina e teve alta com o diagnóstico de angioedema de provável relação com o seu antihipertensor (candesartan + hidroclorotiazida), que foi substituído por amlodipina (5mg id).

Na consulta de reavaliação na USF (13/12/2013) apresentava persistência da sintomatologia, tendo desta vez mencionado também a presença de dispneia para médios esforços e epigastralgias ocasionais, que se 


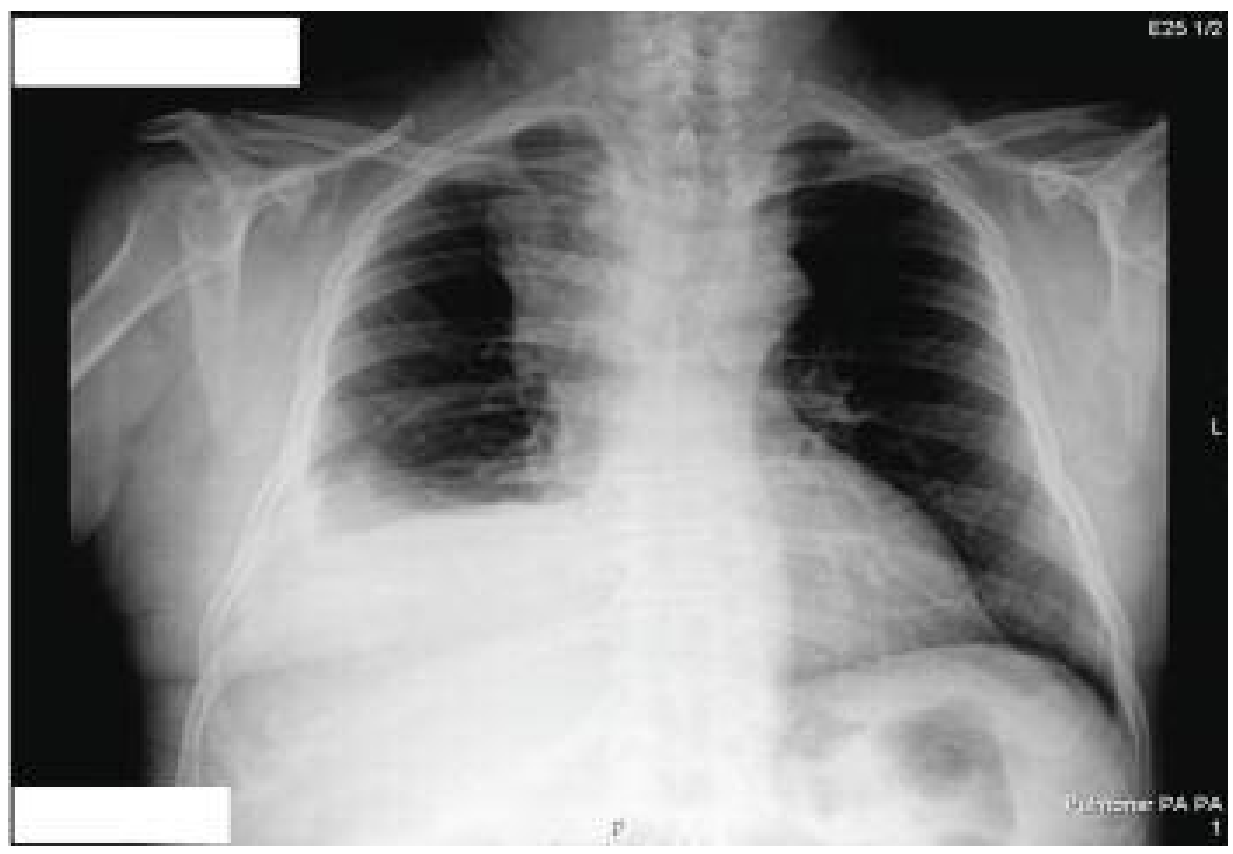

Figura 1. Telerradiografia torácica de fevereiro de 2014. São visíveis o apagamento do ângulo costofrénico direito e o alargamento marcado do mediastino superior.

agravavam após as refeições. Negava tosse, dispneia em repouso, ortopneia, dispneia paroxística noturna e dor pré-cordial, assim como náuseas, vómitos e pirose. Ao exame físico apresentava-se apirético, normotenso, sem adenomegalias palpáveis, alterações auscultatórias e turgescência venosa jugular, tiroide não palpável, com edema facial já conhecido e discreto edema bimaleolar, mas sem edema noutras localizações. Na avaliação do abdómen apenas se detetou desconforto à palpação profunda do epigastro. Perante os novos achados, a hipótese diagnóstica previamente assumida foi colocada em questão. Procurou-se despistar patologia cardíaca e tiroideia que pudessem justificar o edema e a dispneia, mas tanto o ecocardiograma como os valores séricos das hormonas tiroideias eram normais. Foi ainda solicitada repetição da EDA e avaliação em consulta de medicina interna para colaboração na investigação etiológica do angioedema e restantes parâmetros não esclarecidos deste caso.

Na realização da EDA (07/01/2014) foi identificada uma úlcera antro-pilórica de grandes dimensões a condicionar deformação gástrica, com coágulos e sangue digerido, razão pela qual foi encaminhado para o SU do hospital de referência. Após realização de hemostase lo- cal e instituição de terapêutica com inibidor da bomba de protões, o doente ficou internado para estabilização clínica durante cerca de uma semana, tendo tido alta com o diagnóstico de HDA no contexto de úlcera gástrica.

No mês seguinte $(05 / 02 / 2014)$ voltou à USF manifestando agravamento do edema facial e da dispneia, tendo o exame objetivo revelado, além dos achados já conhecidos, adenopatias cervicais bilaterais, peri-centimétricas, elásticas, não aderentes aos planos profundos. Não apresentava sintomatologia ou alterações ao exame objetivo sugestivas de quadro infeccioso. Na ausência de sintomas B e de hepatoesplenomagalia, e dadas as características aparentemente insuspeitas das adenomegalias, não foi levantada a hipótese de doença linfoproliferativa, tendo sido feita nova referenciação para consulta de medicina interna, uma vez que o pedido anterior tinha sido recusado por alegada informação clínica insuficiente.

Após cerca de 15 dias (26/02/2014), recorreu novamente ao SU hospitalar, desta vez por dispneia em repouso, tosse produtiva com expetoração amarelada e dor pleurítica à direita. Mantinha edema facial e apresentava, à auscultação pulmonar, diminuição do murmúrio vesicular à direita. A investigação complementar, realizada com telerradiografia do tórax e estudo analítico, revelou um alargamento do mediastino superior e hipotransparência na base pulmonar direita (Figura 1), assim como valores de $\mathrm{Hb}$ no limite inferior do normal. Foi observado por pneumologia, que solicitou TC cérvico-torácica por suspeita de SVCS, exame este que confirmou a existência de um conglomerado adenopático mediastínico condicionando obstrução da veia cava superior. Já em regime de internamento no serviço de medicina interna foi efetuada uma biópsia 


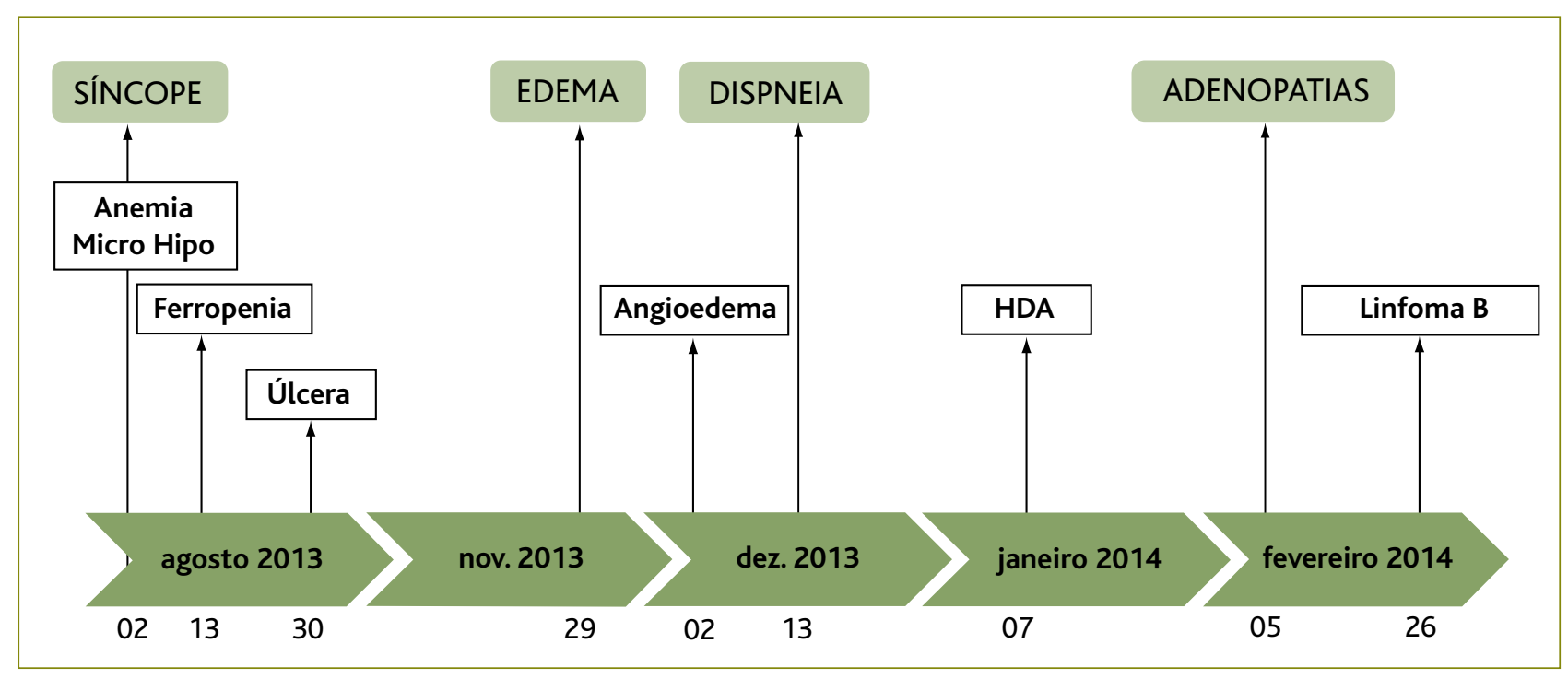

Figura 2. Cronograma.

incisional de adenopatia cervical, cujo resultado histológico se revelou compatível com linfoma B periférico difuso de grandes células. Após terapêutica com diuréticos e corticoterapia, o doente apresentou melhoria sintomática e teve alta orientado para consulta de grupo. Efetuou o primeiro ciclo de quimioterapia em ambulatório.

Os dados mais recentes (19/09/2014), provenientes da consulta de hemato-oncologia, apontavam para o diagnóstico de linfoma não-Hodgkin folicular, transformado em linfoma B difuso de grandes células no estádio IV-A. O doente efetuou, ainda, biópsia de medula óssea que revelou infiltração medular, TC cérvico-torácica que demonstrou regressão das adenopatias após seis ciclos de quimioterapia e biópsias das paredes do estômago que excluíram sinais de malignidade, apesar do espessamento das paredes gástricas de natureza incerta. A sequência cronológica dos acontecimentos é representada na Figura 2.

\section{COMENTÁRIO}

O presente caso clínico vem demonstrar o desafio diagnóstico que as doenças linfoproliferativas podem traduzir, dada a diversidade de quadros clínicos em que se podem apresentar. Estas patologias exigem um elevado índice de suspeição e uma articulação multidis- ciplinar eficiente para o estabelecimento do seu diagnóstico de forma atempada.

O pensamento do especialista em medicina geral e familiar, abrangente e holístico, torna-o capaz de relacionar e integrar todos os achados dos diferentes aparelhos e sistemas, inserindo-os no contexto biopsicossocial e cultural do doente. No entanto, as limitações de caráter logístico inerentes aos cuidados de saúde primários (como a menor acessibilidade a alguns exames complementares e a menor acessibilidade à opinião diferenciada) tornam essencial a existência de uma boa articulação com os cuidados de saúde secundários, que é, por vezes, pouco eficiente e célere, estando sujeita a equívocos de comunicação e a contratempos burocráticos que podem atrasar a orientação dos casos. No presente caso podem levantar-se alguns problemas de natureza organizacional, nomeadamente a articulação difícil com a especialidade de medicina interna (os pedidos de colaboração ineficientes por via informática) e a falta de comunicação intra-hospitalar entre especialidades (note-se que, na altura em que o doente foi internado por HDA em cirurgia geral, não foi descrito qualquer pedido de avaliação do edema facial por outra especialidade).

Do ponto de visto clínico, alguns aspetos deste caso merecem especial destaque: o diagnóstico complicado 
das doenças linfoproliferativas, a etiologia controversa da anemia e o diagnóstico diferencial entre angioedema e SVCS.

Os sintomas mais comumente atribuídos às neoplasias hematológicas são os chamados sintomas B - febre, sudorese noturna e perda ponderal -, que o paciente não apresentava. No entanto, é de salientar que tais sintomas apenas estão presentes em cerca de 28 a $33 \%$ dos linfomas não-Hodgkin mais comuns. ${ }^{6}$ Além destes, outros achados podem estar presentes neste grupo de doenças e merecem investigação adicional e referenciação, como fadiga, prurido generalizado, dispneia, hemorragias gastrointestinais ou outras, infeções de repetição, dor óssea, dor abdominal, linfadenopatias e esplenomegalia. ${ }^{7}$ Destes, o doente apenas apresentava sintomas de dispneia e linfadenopatias (que surgiram já tarde no curso clínico da doença), além da HDA, cujo enquadramento na história do linfoma não é claro. Apesar das perdas hemáticas gastrointestinais serem comuns nas apresentações de certos linfomas (incluindo o linfoma B difuso de grandes células), a úlcera gástrica inicialmente identificada não apresentava sinais de malignidade no exame histológico, assim como não apresentaram as biópsias gástricas efetuadas mais tarde nas consultas de hemato-oncologia. No entanto, está descrito um espessamento das paredes gástricas não explicado. A este respeito, a principal questão que se levanta é se a anemia arrastada que o doente apresentava (nunca completamente resolvida) estaria relacionada apenas com as perdas gástricas associadas à úlcera, contribuindo como fator confundidor no desenrolar deste caso clínico ou se, para a sua etiologia, também contribuiu a doença linfoproliferativa. Sabe-se que as doenças oncológicas, mais especificamente as dos tecidos linfáticos, cursam frequentemente com anemia, seja esta causada por infiltração medular, consumo metabólico ou sequestro esplénico. É possível que a descida da $\mathrm{Hb}$ estivesse relacionada tanto com as perdas hemáticas como com o linfoma no seu estádio inicial. O facto de existir uma explicação evidente para essa descida da $\mathrm{Hb}$ fez esquecer outras causas que podiam contribuir igualmente para a anemia.

Tornou-se, pois, complicado, aos olhos do MF, suspeitar de uma doença linfoproliferativa durante as primeiras consultas. Além disso, estava descrita numa das primeiras idas ao SU, uma telerradiografia torácica (à qual o MF não teve acesso) dentro dos parâmetros da normalidade. Este facto não levou a suspeitar da existência de patologia compressiva no mediastino.

A dificuldade em fazer o diagnóstico diferencial entre angioedema e SVCS revelou-se crucial no desenvolvimento do caso clínico. Uma das causas de angioedema é a terapêutica medicamentosa, sendo mais frequente com os inibidores da enzima de conversão da angiotensina (IECA) e com os anti-inflamatórios não esteróides (AINE), embora também estejam descritas situações de angioedema reativo aos antagonistas dos recetores da angiotensina (ARA) - raciocínio que motivou a suspensão do candesartan. $\mathrm{O}$ angioedema pode ser confundido ou estar relacionado com várias entidades, como dermatites de contacto, celulite, erisipela, linfedema, rosácea, doenças autoimunes como a síndroma de Sjögren e artrite reumatóide, doença de Graves ou doenças tiroideias que envolvam estados mixedematosos e, de particular interesse para este caso, a SVCS. ${ }^{8}$ De facto, estão descritas na literatura situações similares de confusão diagnóstica entre SVCS e angioedema, ${ }^{9-12}$ pelo que ambos devem ser equacionados quando o MF se depara com situações de edema facial. O exame de maior interesse na distinção entre estes dois quadros é a TC torácica, que não deve ser considerada em todas as situações de edema facial, mas apenas nos casos suspeitos e de resistência à terapêutica. Se requisitada precocemente durante o curso clínico da doença tem o potencial de alterar o prognóstico da mesma.

Em suma, dois aspetos que terão condicionado de forma desfavorável o curso deste caso foram a confusão diagnóstica entre angioedema e SVCS e a não prescrição de forma mais precoce da TC torácica. Outro ponto importante foi a dificuldade de articulação com as consultas da especialidade de medicina interna. Estes aspetos devem ser retidos de modo a que se possa aprimorar a efetividade diagnóstica em casos semelhantes. O MF foi o médico omnipresente e o apoio mais próximo, ao qual o doente recorreu repetidamente, procurando, de alguma forma, a solução para o seu sofrimento. Permanecerá sempre a dúvida de qual seria o prognóstico do doente caso o linfoma tivesse sido detetado mais cedo; no entanto, a presença constante de um profissional de saúde que acolheu o doente 


\section{durante todo o percurso clínico foi imprescindível para a manutenção da esperança de resolução do quadro.}

\section{AGRADECIMENTOS}

Os autores agradecem à Dra. Manuela Archer pela colaboração preciosa na realização do presente trabalho.

\section{REFERÊNCIAS BIBLIOGRÁFICAS}

1. Li HH. Angioedema. Medscape; 2015 Apr 1 [cited 2015 May 7]. Available from: http://emedicine.medscape.com/article/135208-overview

2. Madsen F, Attermann J, Linneberg A. Epidemiology of non-hereditary angioedema. Acta Derm Venereo. 2012;92(5):475-9.

3. Muller BA. Urticaria and angioedema: a practical approach. Am Fam Physician. 2004;69(5):1123-8.

4. Rice TW, Rodriguez RM, Light RW. The superior vena cava syndrome: clinical characteristics and evolving etiology. Medicine (Baltimore). 2006;85(1):37-42.

5. Wilson LD, Detterbeck FC, Yahalom J. Superior vena cava syndrome with malignant causes. N Engl J Med. 2007;356:1862-9.

6. Hematopoietic disorders. In Longo DL, Fauci AS, Kasper DL, Hauser SL, Jameson JL, Loscalzo J. Harrison's principles of internal medicine. 18th ed. McGraw-Hill; 2011. p. 919-35. ISBN 9780071771962

7. Clinical Governance Research and Development Unit, Department of Health Sciences, University of Leicester. Referral guidelines for suspected cancer in adults and children: NICE clinical guidelines. London: Royal College of General Practitioners; 2005 [cited 2015 May 7]. Available from: http://www.ncbi.nlm.nih.gov/books/NBK45773/
8. Zuraw B, Bingham III CO. An overview of angioedema: clinical features, diagnosis and management. UpToDate; 2015 [cited 2015 May 8]. Available from: http://www.uptodate.com/contents/an-overview-ofangioedema-clinical-features-diagnosis-and-management\#H22

9. Angevine $C D$, Condemi JJ.The facial edema of vena cava syndrome mistaken for angioneurotic edema. J Allergy. 1966;38(5):308-14.

10. Pinto N, Rosa S, Vargas F, Pinto PL. Do processo linfoproliferativo ao angioedema. Rev Port Imunoalergol. 2014;XXII Suppl 1:59. Portuguese

11. Burgdorff T, Douwes KE, Bogenrieder T, Szeimies RM, Hohenleutner U, Landthaler $M$, et al. Superior vena cava syndrome: an important differential diagnosis in patients with facial edema. Acta Derm Venereol. 2001;81(3):205-6.

12. Terasaki K, Ohkubo K, Kanekura T. Facial edema as a clue to superior vena cava syndrome associated with lung cancer. Eur J Dermatol. 2012;22(4):546-7.

\section{CONFLITO DE INTERESSES}

Os autores declaram a inexistência de qualquer tipo de conflito de interesse envolvido no presente trabalho, bem como a inexistência de qualquer tipo de financiamento externo.

\section{ENDEREÇO PARA CORRESPONDÊNCIA}

João Vieira Antunes

Rua do Chaimite, n. 344 | 4435 - 025 Rio Tinto

E-mail: joaopeantunes@gmail.com

\section{Recebido em 15-12-2014}

Aceite para publicação em 20-06-2015 


\section{ABSTRACT}

\section{ANGIOEDEMA OR SUPERIOR VENA CAVA SYNDROME: A CASE-REPORT}

Introduction: The family doctor often sees signs and symptoms of an apparently benign nature that may turn out to be part of more severe disease. This case presents the common finding of facial edema that was interpreted as angioedema, when the underlying disease was the superior vena cava syndrome (SVCS).

Case description: A 61-year-old man consulted his family doctor because of an episode of syncope. He was sent to the hospital emergency department, where vasovagal syncope was diagnosed and a microcytic hypochromic anemia was found. Subsequent investigations revealed iron deficiency anemia and a gastric antral ulcer. Three months later, he returned to the Family Health Unit with facial edema and was treated with an antihistamine. Because of persistence of his complaints, he consulted at the hospital emergency department, where angioedema was diagnosed. One month later, he was admitted to the hospital for treatment of gastrointestinal bleeding from the gastric ulcer. He later returned to the hospital emergency department with respiratory symptoms. A chest radiograph revealed enlargement of the superior mediastinum. Following admission to hospital, the diagnosis of a B-cell lymphoma was made after an incisional biopsy of an enlarged cervical lymph node.

Comment: This case illustrates the diagnostic challenge presented by lymphoproliferative disorders. Distinguishing between angioedema and superior vena cava syndrome was a central challenge. Cases of diagnostic confusion between these two entities have been described in the literature. Questions related to the complexity of clinical presentations and the difficulties experienced by the family doctor in the management of this case are discussed.

Keywords: Lymphoma; Angioedema; Superior vena cava syndrome. 